\title{
Predicting Discourse Trees from Transformer-based Neural Summarizers
}

\author{
Wen Xiao, Patrick Huber, Giuseppe Carenini \\ Department of Computer Science \\ University of British Columbia \\ Vancouver, BC, Canada, V6T 1Z4 \\ \{xiaowen3, huberpat, carenini\}@cs.ubc.ca
}

\begin{abstract}
Previous work indicates that discourse information benefits summarization. In this paper, we explore whether this synergy between discourse and summarization is bidirectional, by inferring document-level discourse trees from pre-trained neural summarizers. In particular, we generate unlabeled RST-style discourse trees from the self-attention matrices of the transformer model. Experiments across models and datasets reveal that the summarizer learns both, dependency- and constituencystyle discourse information, which is typically encoded in a single head, covering long- and short-distance discourse dependencies. Overall, the experimental results suggest that the learned discourse information is general and transferable inter-domain ${ }^{1}$.
\end{abstract}

\section{Introduction}

Extractive summarization is a common and important task within the area of Natural Language Processing (NLP), which can be useful in a multitude of diverse real-life scenarios. Current extractive summarizers typically use exclusively neural approaches, in which the importance of extracted units (i.e., sentences or clauses) and relationship between them are learned by the model from a large amount of data (e.g., Liu and Lapata (2019b)).

Inspired by previous work in pre-neural times, indicating that discourse information, especially discourse trees according to the Rhetorical Structure Theory (RST) (Mann and Thompson, 1988), can benefit the summarization task (Marcu, 1999), several very recent neural summarizers have tried to explicitly encode discourse information to support summarization. Overall, it seems that adding these encodings, consistent with pre-neural results, is beneficial. In particular, injecting discourse has been shown to either improve performance

\footnotetext{
${ }^{1}$ The code can be found in https://github.com/ Wendy-Xiao/summ_guided_disco_parser
}

on the extractive summarization task itself (Xu et al., 2020), or allow for a substantial reduction in the number of the summarizer's parameters, while keeping competitive performance (Xiao et al., 2020).

The central hypothesis we are exploring in this paper is whether the synergy between discourse parsing and summarization is bidirectional. In other words, we examine if summarization is a useful auxiliary task to infer discourse structures. Liu et al. (2019b) performed a preliminary investigation of this conjecture, showing that structural information can be inferred from attention mechanisms while training a neural model on auxiliary tasks. However, they did not perform any comparison against ground-truth discourse trees. Further, recent work showed that discourse trees implicitly induced during training are oftentimes trivial and shallow, not representing valid discourse structures (Ferracane et al., 2019).

In this paper, we address these limitations by explicitly exploring the relationship between summarization and discourse parsing through the inference of document-level discourse trees from pretrained summarization models, comparing the results against ground-truth RST discourse trees. Besides Liu et al. (2019b), our idea and approach are inspired by recent works on extracting syntactic trees from pre-trained language models (Wu et al., 2020) or machine translation approaches (Raganato and Tiedemann, 2018), as well as previous work on knowledge graph construction from pre-trained language models (Wang et al., 2020). Specifically, we generate full RST-style discourse trees from self-attention matrices of a pre-trained transformerbased summarization model. We use three different tree-aggregation approaches (CKY (Jurafsky and Martin, 2014), Eisner (Eisner, 1996) and CLE (Chu and Liu, 1965; Edmonds, 1967)), generating a set of constituency and dependency trees representing diverse discourse-related attributes. 
Our proposal is thereby addressing one of the key limitations in discourse parsing, namely the lack of large training corpora. We aim to overcome this limitation by generating a large number of reasonable quality discourse trees from a pre-trained summarization model, similar in spirit to what Huber and Carenini (2020) did with sentiment. Admittedly, the discourse information captured with our approach is summarization task-specific, however, our generated discourse treebank can be combined with further task-dependent treebanks (e.g. from sentiment) to train more powerful discourse parsers in a multitask framework.

Generally speaking, the ability to infer discourse trees as a "by-product" of the summarization task can also be seen as a form of unsupervised discourse parsing, where instead of leveraging pretrained language models like in Kobayashi et al. (2019), we exploit a pre-trained neural summarizer

We empirically evaluate our method on three datasets with human RST-style annotations, covering different text genres. Multiple experiments show that the summarization model learns discourse information implicitly, and that more dependency information are captured, compared to structural (i.e., constituency) signals. Interestingly, an additional exploration of the attention matrices of individual heads suggests that, for all models, most of the discourse information is concentrated in a single head, and the best performing head is consistent across all datasets. We further find that the dependency information learned in the attention matrix covers long distance discourse dependencies. Overall, the results are consistent across datasets and models, indicating that the discourse information learned by the summarizer is general and transferable inter-domain.

\section{Related Work}

Rhetorical Structure Theory (RST) (Mann and Thompson, 1988) is one of the most popular theories of discourse, postulating that a document can be represented as a constituency tree, where leaves are clause-like Elementary Discourse Units (EDUs), and internal nodes combine their respective children by aggregating them into a single, joint constituent. Each internal node also has a nuclearity attribute ${ }^{2}$, representing the local importance of their direct child-nodes in the parent context from the set of \{Nucleus-Nucleus,

\footnotetext{
${ }^{2}$ In this paper we do not consider rhetorical relations.
}

Nucleus-Satellite, Satellite-Nucleus \}. "Nucleus" child-nodes thereby generally play a more important role when compared to a "Satellite" child-node. Although standard RST discourse trees are encoded as constituency trees, they can be converted into dependency trees with near isomorphic transformations. In this work, we infer both, constituency and dependency trees.

Over the past decades, RST discourse parsing has been mainly focusing on supervised models, typically trained and tested within the same domain using human annotated discourse treebanks, such as RST-DT (Carlson et al., 2002), Instruction-DT (Subba and Di Eugenio, 2009) or GUM (Zeldes, 2017). The intra-domain performance of these supervised models has consistently improved, with a mix of traditional models by Joty et al. (2015) and Wang et al. (2017), and neural models (Yu et al., 2018) reaching state-of-the-art (SOTA) results. Yet, these approaches do not generalize well inter-domain (Huber and Carenini, 2020), likely due to the limited amount of available training data.

Huber and Carenini (2019) recently tackled this data-sparsity issue through automatically generated discourse structures from distant supervision, showing that sentiment information can be used to infer discourse trees. Improving on their initial results, Huber and Carenini (2020) published a large-scale, distantly supervised discourse corpus (MEGA-DT), showing that a parser trained on such treebank delivers SOTA performance on the more general interdomain discourse parsing task. In this paper, we also tackle the data sparsity problem in discourse parsing, however, using a significantly different approach. First, instead of relying on sentiment, we leverage the task of extractive summarization. Second, instead of a method for distant supervision, we propose an unsupervised approach.

The area of unsupervised RST-style discourse parsing has been mostly underlooked in the past, with recent neural approaches either taking advantage of pre-trained language models to predict discourse (Kobayashi et al., 2019) or using pre-trained syntactic parsers and linguistic knowledge (Nishida and Nakayama, 2020) to infer discourse trees in an unsupervsied manner. Similarly. our proposal only relies on a pre-trained neural summarization model to generate discourse trees.

Recent neural summarization models are typically based on transformers (Liu and Lapata, 2019a; Zhang et al., 2019). One advantage of these mod- 
els is that they learn the relationship between input units explicitly using the dot-product self-attention, which allows for some degree of exploration of the inner working of these complex and distributed models. Here, we investigate if the attention matrices of a transformer-based summarizer effectively capture discourse information (i.e., how strongly EDUs are related) and therefore can be used to derive discourse trees for arbitrary documents.

Marcu (1999) pioneered the idea to directly apply RST-style discourse parsing to extractive summarization, and empirically showed that RST discourse information can benefit the summarization task, by simply extracting EDUs along the nucleus path. This initial success was followed by further work on leveraging discourse parsing in summarization, including McDonald (2007), Hirao et al. (2013), and Kikuchi et al. (2014). More recently, the benefits of discourse for summarization have also been confirmed for neural summarizers, e.g. in Xiao and Carenini (2019) and Cohan et al. (2018), using the structure of scientific papers (i.e. sections), and in Xu et al. (2020), successfully incorporating RST-style discourse and co-reference information in the BERTSUM summarizer (Liu and Lapata, 2019b).

In contrast to previous approaches demonstrating how discourse can enhance summarization performance, we have recently shown that discourse enables the specification of simpler neural summarizers, without affecting their performance (Xiao et al., 2020). In particular, by using a fixed discoursebased attention they achieve competitive results compared to learnable dot-product self-attention mechanisms, as used in the original transformer model. Inspired by these findings, suggesting that transformer-based summarization models learn effective discourse representations, we explore if useful discourse structures can be inferred from learnt transformer self-attention weights.

Admittedly, Liu and Lapata (2018) and Liu et al. (2019b) presented preliminary work on inferring discourse structures from attention mechanisms, while training a neural model on auxiliary tasks, like text classification and summarization. However, they did not perform any comparison against ground-truth discourse trees as we do here. More importantly, we employ a more explicit approach to infer discourse structures, not as part of the learning process, but extracting the structures after the summarization model is completely trained and applied to new documents.

While our focus is on discourse, extracting syntactic constituency and dependency trees from transformer-based models has been recently attempted in both, machine translation and language modelling. In machine translation, Mareček and Rosa (2019) and Raganato and Tiedemann (2018) show that trained translation models can capture syntactic information within their attention heads, using the CKY and CLE algorithms, respectively. In pre-trained language models, Wu et al. (2020) propose a parameter-free probing method to construct syntactic dependency trees based on a pretrained BERT model, only briefly elaborating on possible applications to discourse. In contrast to our work, they do not directly use attention heads, but instead build an impact matrix based on the distance between token representations. Furthermore, while their BERT-based model cannot deal with long sequences, our two-level encoder can effectively deal with sequences of any length, which is critical in discourse.

\section{Our Model}

\subsection{Framework Overview}

Our main goal is to show the ability of a previously trained summarization model to be directly applied to the task of RST-style discourse parsing. Along this line, we explore the relationship between information learned by the transformer-based sumarizer and the task of discourse parsing. We leverage the synergies between units learned in the transformer model by following Xiao et al. (2020), previously proposing the use of a transformer documentencoder on top of a pretrained BERT EDU encoder. This standard summarization model is presented in Figure 1 (left). In the transformer-based document encoder, each head internally contains a selfattention matrix, learned during the training of the summarization model, representing the relationship between EDUs (Figure 1 (center)). In this paper, we analyze these learned self-attention matrices, not only to confirm our intuition that they contain relevant discourse information, but also to computationally exploit such information for discourse parsing. We therefore generate a set of different (constituency/dependency) discourse trees from the self-attention matrices, focusing on different attributes of discourse, as shown in Figure 1 (right). Our generated constituency trees only reveal the discourse tree structure without additional nucle- 

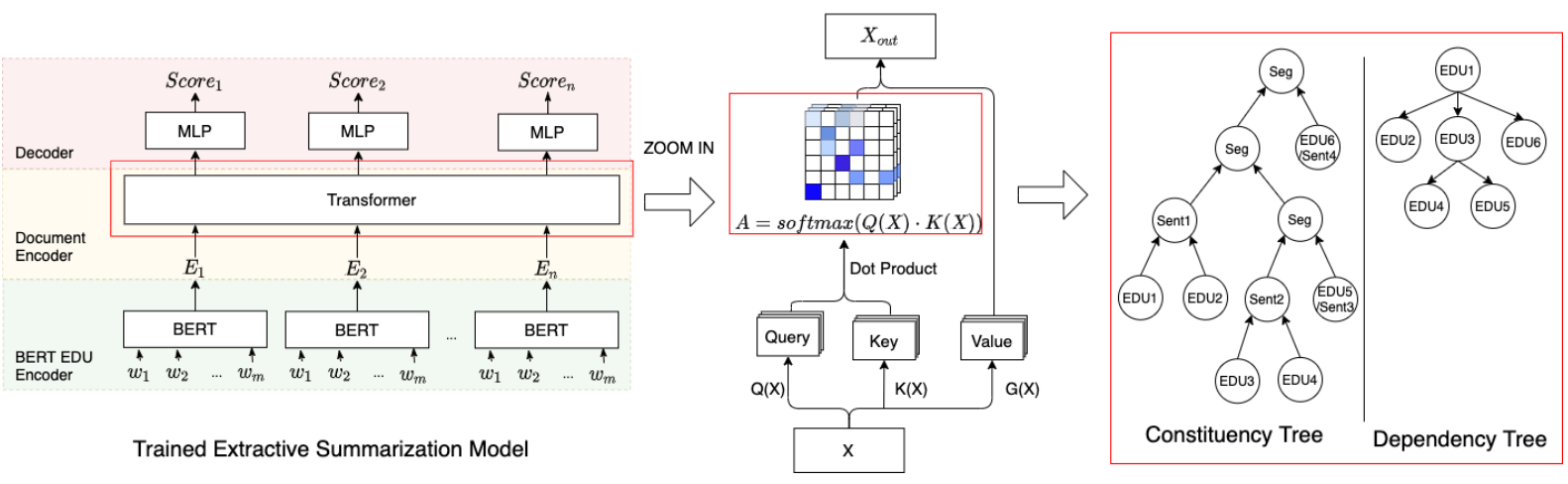

Figure 1: The pipeline of our whole method.

arity and relation attributes. More interestingly, we complement the constituency interpretation of the self-attention by additionally inferring a dependency tree, also partially guided by discourse structures, but mostly driven by the RST nuclearity attribute, which is shown to be more related to the summarization task where the importance of the different text spans is critical (Hirao et al., 2013). We present and discuss the different parsing algorithms to extract discourse information from the self-attention matrix next.

\subsection{Parsing Algorithms}

Formally, for an input document $D=\left\{u_{1}, . ., u_{n}\right\}$ with $n$ EDUs, each attention head returns an attention matrix $A \in \mathbf{R}^{n \times n}$ where entry $A_{i j}$ contains a score measuring how much the $i$-th EDU relies on the $j$-th EDU. Given those bidirectional scores defining the relationship between every two EDUs in a document, we build a tree such that EDU pairs with higher reciprocal attention scores are more closely associated in the resulting tree. In the constituency case, this means that EDUs with higher mutual attention should belong to sub-trees on lower levels of the tree, while in the dependency case this implies that the path between such EDUs should contain less intermediate nodes. In essence, these requirements can be formalized as searching for the tree within the set of possible trees, which maximizes a combined score.

\subsubsection{Constituency Tree (C-Tree) Parsing}

To generate a constituency tree from the attention matrix, we follow a large body of previous work in discourse parsing (e.g., Joty et al. (2015)), where constituency discourse trees are generated using the CKY algorithm (Jurafsky and Martin, 2014). Specifically, we fill a $n \times n$ matrix $P \in \mathbf{R}^{n \times n}$ generating the optimal tree in bottom-up fashion using the dynamic programming approach according to:

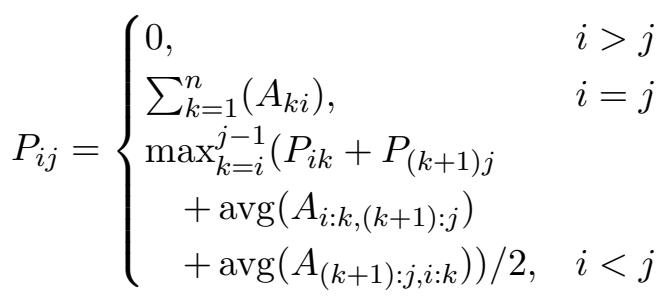

where $P_{i j}$ with $i=j$ contains the overall importance of EDU $i$, computed as the attention paid by others to unit $i$. $P_{i j}$ with $i<j$ represents the score of the optimal sub-tree spanning from EDU $i$ to EDU $j$. We select the best combination of sub-trees $k$, such that the sum of the left subtree spanning $[i: k]$ and the right one spanning $[(k+1): j]$, along with the average score of connections between the two sub-trees is maximized.

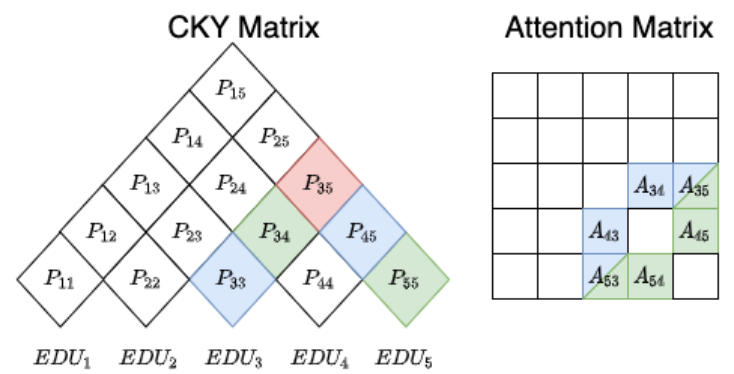

Figure 2: Example of CKY constituency parsing.

For example, to pick the structure of the sub-tree spanning EDUs [3 : 5] (see Fig. 2), we need to decide between the potential sub-tree aggregation of $((34) 5)$ and $(3(45))$. The respective scores are computed based on the scores in green and blue blocks in both the CKY and the Attention Matrices. Following this algorithm, two sub-trees with a high attention score between them tend to be combined on lower levels of the tree, indicating they are more related in the discourse tree.

Besides the standard CKY algorithm described 
(a)

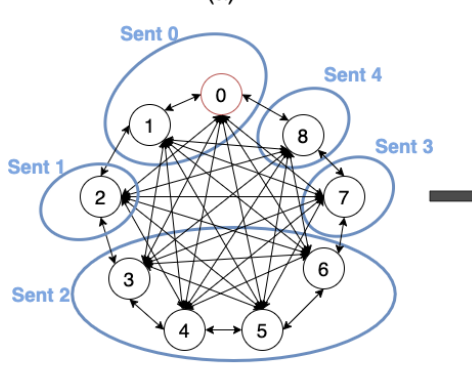

(b)

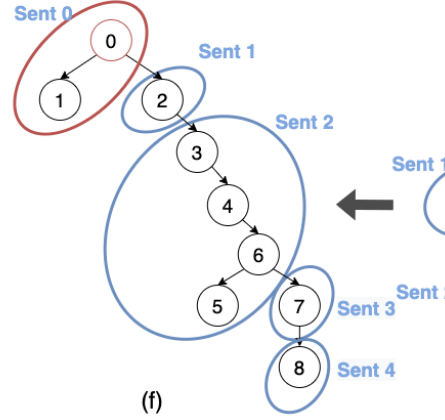

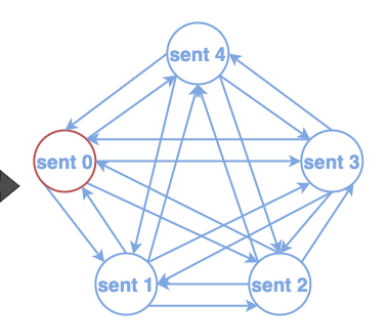

(c)
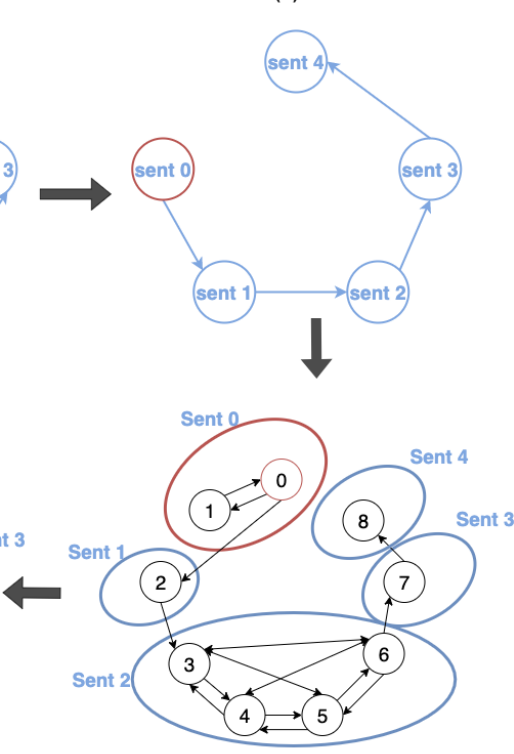

(d)

Figure 3: Chu-Liu-Edmonds Algorithm with sentence constraints

above, we also explore a hierarchical CKY approach with sentence and paragraph constraints. Specifically, we do not aggregate $P_{i j}$ if the span $[i: j]$ crosses a sentences boundary where either sentence is incomplete. In the previous example, if $E D U_{3}$ and $E D U_{4}$ were in the same sentence, even if the score of the blue aggregation candidate was higher, we would choose the green sub-tree aggregation. Plausibly, this hierarchical approach will perform better, since the ground-truth treebanks mostly contain sentences and paragraphs that are covered by a complete discourse sub-trees.

\subsubsection{Dependency Tree (D-Tree) Parsing}

For the dependency tree generation, we use the Eisner (Eisner, 1996) and Chu-Liu-Edmonds algorithm (Chu and Liu, 1965; Edmonds, 1967) to generate projective and non-projective dependency trees, respectively ${ }^{3}$. First, we convert the attention matrix $A$ into a fully connected graph $G=(N, E)$, where $N$ contains all the EDUs, and $e_{i j}$, indicating how much the $i$-th EDU influences the $j$-th EDU, corresponds to $A_{j i}$, which is the attention that the $j$ th EDU pays to the $i$-th EDU. Based on this graph, we apply the following algorithms:

Eisner Algorithm: We apply this dynamic programming algorithm to generate projective dependency trees. Thereby, we build a matrix $P \in$ $\mathbf{R}^{n \times n \times 2 \times 2}$, in which the first and second dimen-

\footnotetext{
3"Mixed" approaches, dealing with mildly non-projective trees (Kuhlmann and Nivre, 2006), are left for future work.
}

sions contain the start and end indexes of sub-trees, similar to the CKY algorithm; while the third and fourth dimensions indicate whether the head is the start or the end unit, and whether the sub-tree is completed. As done for constituency parsing, we also use a hierarchical version of Eisner's algorithm, in which we restrict inter-sentence connections for incomplete sentence trees. Since the Eisner algorithm can only generate projective dependency trees, it will be inaccurate for documents with a non-projective discourse structure.

Chu-Liu-Edmonds (CLE) Algorithm: Originally proposed as a recursive approach to find the maximum spanning tree of a graph given its root, CLE can generate non-projective trees. In the unconstrained case, we simply follow the standard CLE algorithm, selecting the EDU with the highest importance score, computed similar to Sec. 3.2.1, i.e. root $=\operatorname{argmax}_{i} \sum_{k=1}^{n}\left(A_{k i}\right)$, as the root. From there, the algorithm selects the "optimal edges", i.e. the maximum in-edges for each node except the root, breaking the cycles recursively.

Again, as we did for CKY and Eisner, we also apply the additional sentence constraint. Unlike for the dynamic programming approaches, which build the trees in a bottom-up fashion and can directly be constrained to avoid cross-sentence aggregations of incomplete sentences, we need to substantially modify CLE to allow for sentence constraints.

In particular, we first build a sentence graph $G^{s}=\left\{N^{s}, E^{s}\right\}$ from the EDU graph (Figure 3 
(b)), in which $e_{S D}^{s}=\operatorname{avg}_{s \in S, d \in D} e_{s d}$, and record the maximum edge corresponding to the edge between sentences, i.e. $\operatorname{argmax}_{s \in S, d \in D} e_{s d}$. After that, we use the CLE algorithm within the sentence containing the root EDU as the root sentence to find the maximum spanning tree in $G^{s}$ (Figure 3 (c)). We then add the corresponding EDU edges to the final tree (Figure $3(\mathrm{~d})$ ). For example, the edge $\left(s_{0}, s_{1}\right)$ in $G^{s}$ corresponds to the EDU edge $\left(e_{0}, e_{2}\right)$ in $G$. Next, we treat nodes with incoming edges from other sentences as the root of the sentence itself and run the CLE algorithm within each sentence (Figure 3 (e)). The final tree (Figure 3 (f)) is eventually formed as the combination of intersentence edges derived in sentence graph $G_{s}$ and intra-sentence edges found within each sentence.

\section{Experiments and Analysis}

\subsection{The Summarization Task}

In order to show the generality of the discourse structures learned in the summarization model, we train our summarizer across a variety of datasets and hyper-parameter settings. More specifically, we train on two separate, widely-used news corpora - CNN Daily Mail (CNNDM) (Nallapati et al., 2016) and NYT (Sandhaus, 2008) -, as well as under three hyper-parameter settings with different numbers of layers and attention heads: (a) A simple model with 2 layers and a single head. (b) 6 layers with 8 heads each, proposed in the original transformer model(Vaswani et al., 2017). (c) 2 layers with 8 heads each, constituting a middle ground between the previous two settings. By considering two corpora (CNNDM and NYT) and the three settings, we train six models, which we call: CNNDM-2-1, CNNDM-6-8, CNNDM-2-8, NYT2-1, NYT-6-8, NYT-2-8 ${ }^{4}$.

\subsection{Discourse Datasets}

The quality of the attention-generated trees is assessed on three discourse datasets (see Table 1).

RST-DT is the largest and most frequently used RST-style discourse treebank (Carlson et al., 2002), containing news articles from the Wall Street Journal. Since this is the genre of both our summarization training corpora, the experiments testing on this dataset are intra-domain.

Instruction-DT contains documents in the home-repair instructions domain (Subba and Di Eugenio, 2009). We categorize the experiments on

\footnotetext{
${ }^{4}$ Complete evaluation results for all six models are presented in Appendix A.
}

this dataset as cross-domain.

GUM contains documents from eight domains including news, interviews, academic papers and more (Zeldes, 2017). Since the GUM corpus is multi-domain, the performance on this dataset will reveal the generalizability of generated trees in a broader sense.

\begin{tabular}{c|c|c|c|c} 
Dataset & \# Docs & \#EDU/doc & \#Sent/doc & \#words/doc \\
\hline RST-DT & 385 & 56.6 & 22.5 & 549 \\
Instruction & 176 & 32.7 & 19.5 & 318 \\
GUM & 127 & 107 & 45 & 874 \\
\hline
\end{tabular}

Table 1: Key RST-style discourse dataset dimensions.

All three discourse datasets contain ground-truth RST-style consituency trees. While all corpora contain potential non-binary sub-trees, Instruction-DT also includes multi-root documents. To account for these cases, we apply the right-branching binarization following Huber and Carenini (2019). Furthermore, we convert constituency trees with nuclearity into ground truth dependency trees using the algorithm proposed in Li et al. (2014) .

\subsection{Evaluation Metric}

To evaluate how well the generated trees align with ground-truth trees, we use RST Parseval Scores for constituency trees and Unlabeled Attachment Score for dependency trees, measuring the ratio of matched spans and the ratio of matched dependency relations, respectively.

\subsection{Overall Results}

For each model configuration, we run a set of experiments using the average attention matrix across all heads in a layer, i.e. $A_{\text {avg }}=\sum_{h} A^{h} / H$, with $H$ as the number of heads. This initial setup is intended to provide insights into the discourse information learned in each layer.

The results of the three tree-generation algorithms are shown in Table 2, 3 and 4 along with the performance of a random baseline obtained by running the algorithms on 10 random matrices. Here, we present the results of three selected models, limited to the performance of the first two layers for the 6-layer models, to allow for a direct comparison to the 2-layer models ${ }^{5}$. Across evaluations, the layer-wise performance within the same models are rather distinct, indicating that different properties are learned in the layers. This finding is in line with previous work (Liu et al., 2019a), especially

\footnotetext{
${ }^{5}$ Results for all six models can be found in Appendix B.
} 


\begin{tabular}{c|c|r|r|r}
\hline \multirow{2}{*}{ Model } & \multicolumn{2}{|c|}{ No Cons. } & \multicolumn{2}{c}{ Sent Cons. } \\
\cline { 2 - 5 } & Attn\#0 & Attn\#1 & Attn\#0 & Attn\#1 \\
\hline \multicolumn{5}{|c}{ RST-DT } \\
\hline CNNDM-2-1 & 61.2 & 59.7 & 76.2 & 74.6 \\
CNNDM-6-8 & 60.3 & 60.8 & 75.4 & 75.0 \\
NYT-6-8 & $\mathbf{6 2 . 4}$ & 62.2 & 76.7 & 75.6 \\
\hline Random & \multicolumn{5}{c}{$58.6 \pm 0.1$} & $74.1 \pm 0.1$ \\
\hline \multicolumn{5}{c}{ Instruction } \\
\hline CNNDM-2-1 & 61.1 & 59.8 & 71.4 & $\downarrow 70.3$ \\
CNNDM-6-8 & 60.3 & $\mathbf{6 1 . 2}$ & 71.2 & 70.9 \\
NYT-6-8 & $\mathbf{6 1 . 3}$ & 61.3 & 71.3 & $\downarrow 70.0$ \\
\hline Random & $59.5 \pm 0.3$ & $70.5 \pm 0.1$ \\
\hline \multicolumn{5}{|c}{ GUM } \\
\hline CNNDM-2-1 & 58.7 & 57.7 & 72.7 & 71.9 \\
CNNDM-6-8 & 58.9 & 59.3 & 72.4 & $\mathbf{7 2 . 7}$ \\
NYT-6-8 & 59.6 & 59.3 & 72.2 & 71.6 \\
\hline Random & $57.5 \pm 0.1$ & $71.5 \pm 0.2$ \\
\hline
\end{tabular}

Table 2: RST Parseval Scores of generated constituency trees on the three datasets, expressed as 'Avg. \pm Std'. Green means the result is better than Random, and Red along with $\downarrow$ means worse. Results for Random are obtained by applying the parser to random matrices for 10 times. Attn\#0/1 are the first two layers.

given that the performance of each layer is consistent across constituency and dependency parsing outputs for all datasets. Furthermore, the more layers the summarization model contains, the smaller the performance gap between layers becomes. We believe that this could be caused by the discourse information being further spread across different layers. Generally, we observe that models trained on the CNNDM dataset perform better than models trained on the NYT corpus, despite the larger size of the NYT dataset. Plausibly, the superior performance of our models trained on CNNDM potentially reflects a higher diversity within documents in the CNNDM dataset.

Comparing the constituency tree performance in Table 2 against the dependency tree results in Tables 3 and 4, we can clearly see that the improvement of the constituency parsing approach over the random baseline is much smaller than the improvements for the generated dependency parse-trees. Presumably, this larger improvement for the dependency trees is due to the fact that dependency relationships (strongly encoding the nuclearity attribute) are more directly related to the summarization task than the plain structure information. This is in line with previous work on applying dependency trees to the summarization task (Hirao et al., 2013; Xu et al., 2020) and indicates that the learned attention matrices contain valid discourse information.

\begin{tabular}{c|r|r|r|r}
\hline \multirow{2}{*}{ Model } & \multicolumn{2}{|c|}{ No Cons. } & \multicolumn{2}{c}{ Sent Cons. } \\
\cline { 2 - 5 } & Attn\#0 & Attn\#1 & Attn\#0 & Attn\#1 \\
\hline \multicolumn{5}{|c}{ RST-DT } \\
\hline CNNDM-2-1 & 23.7 & $\downarrow 4.8$ & $\mathbf{2 8 . 2}$ & $\downarrow 18.2$ \\
CNNDM-6-8 & $\downarrow 7.9$ & 20.5 & $\downarrow 13.8$ & 27.8 \\
NYT-6-8 & 15.7 & 12.5 & 24.3 & $\downarrow 18.9$ \\
\hline Random & \multicolumn{5}{c}{$11.2 \pm 0.2$} & $20.3 \pm 0.2$ \\
\hline \multicolumn{5}{|c}{ Intruction } \\
\hline CNNDM-2-1 & 31.1 & $\downarrow 4.4$ & 29.3 & $\downarrow 13.5$ \\
CNNDM-6-8 & $\downarrow 8.5$ & 19.5 & $\downarrow 9.9$ & 22.0 \\
NYT-6-8 & 16.2 & $\downarrow 12.1$ & 22.8 & $\downarrow 16.4$ \\
\hline Random & \multicolumn{5}{|c}{$13.1 \pm 0.3$} & $19.3 \pm 0.4$ \\
\hline \multicolumn{5}{|c|}{ GUM } \\
\hline CNNDM-2-1 21.3 & $\downarrow 2.24$ & 27.3 & $\downarrow 16.1$ \\
CNNDM-6-8 & $\downarrow 4.7$ & 15.8 & $\downarrow 11.5$ & 24.80 \\
NYT-6-8 & 12.6 & $\downarrow 9.6$ & 23.4 & $\downarrow 17.1$ \\
\hline Random & \multicolumn{3}{c}{$10.4 \pm 0.2$} & \multicolumn{3}{c}{$19.2 \pm 0.3$} \\
\hline
\end{tabular}

Table 3: Unlabeled Attachment Scores of dependency trees generated by the Eisner algorithm.

\begin{tabular}{c|r|r|r|r}
\hline \multirow{2}{*}{ Model } & \multicolumn{2}{|c|}{ No Cons. } & \multicolumn{2}{c}{ Sent Cons. } \\
\cline { 2 - 5 } & Attn\#0 & Attn\#1 & Attn\#0 & Attn\#1 \\
\hline \multicolumn{5}{|c}{ RST-DT } \\
\hline CNNDM-2-1 & $\mathbf{2 1 . 6}$ & $\downarrow 1.5$ & $\mathbf{2 9 . 3}$ & 19.6 \\
CNNDM-6-8 & 7.3 & 17.3 & $\downarrow 16.1$ & 28.5 \\
NYT-6-8 & 13.7 & 10.6 & 25.0 & 21.1 \\
\hline Random & \multicolumn{6}{c}{$1.7 \pm 0.1$} & $18.7 \pm 0.1$ \\
\hline \multicolumn{5}{c}{ Instruction } \\
\hline CNNDM-2-1 & $\mathbf{2 8 . 1}$ & $\downarrow 2.1$ & $\mathbf{3 7 . 4}$ & 18.1 \\
CNNDM-6-8 & 6.9 & 15.9 & $\downarrow 14.9$ & 25.8 \\
NYT-6-8 & 14.8 & 9.8 & 25.4 & 21.1 \\
\hline Random & $2.9 \pm 0.2$ & $17.9 \pm 0.4$ \\
\hline \multicolumn{5}{|c}{ GUM } \\
\hline CNNDM-2-1 & 19.5 & $\downarrow 0.7$ & $\mathbf{2 8 . 8}$ & 17.9 \\
CNNDM-6-8 & 4.0 & 13.1 & $\downarrow 14.9$ & 25.4 \\
NYT-6-8 & 10.7 & 8.2 & 23.0 & 19.5 \\
\hline Random & $0.9 \pm 0.05$ & $17.0 \pm 0.2$ \\
\hline
\end{tabular}

Table 4: Unlabeled Attachment Scores of dependency trees generated by the CLE algorithm

As for the two approaches to dependency parsing, although Eisner generally outperforms CLE, the improvement over random trees is larger for CLE. We believe that this effect is due to the reduced constraints imposed on the CLE algorithm, which is not limited to generate projective trees.

Considering all three methods, the results of the CLE-generated dependency tree seem most promising. A possible explanation is that both CKY and Eisner build the discourse tree in a bottom-up fashion with dynamic programming. This way, only local information is used on lower levels of the tree. On the other hand, the CLE algorithm uses global information, potentially more aligned with the summarization task, where all EDUs are considered to predict importance scores. 


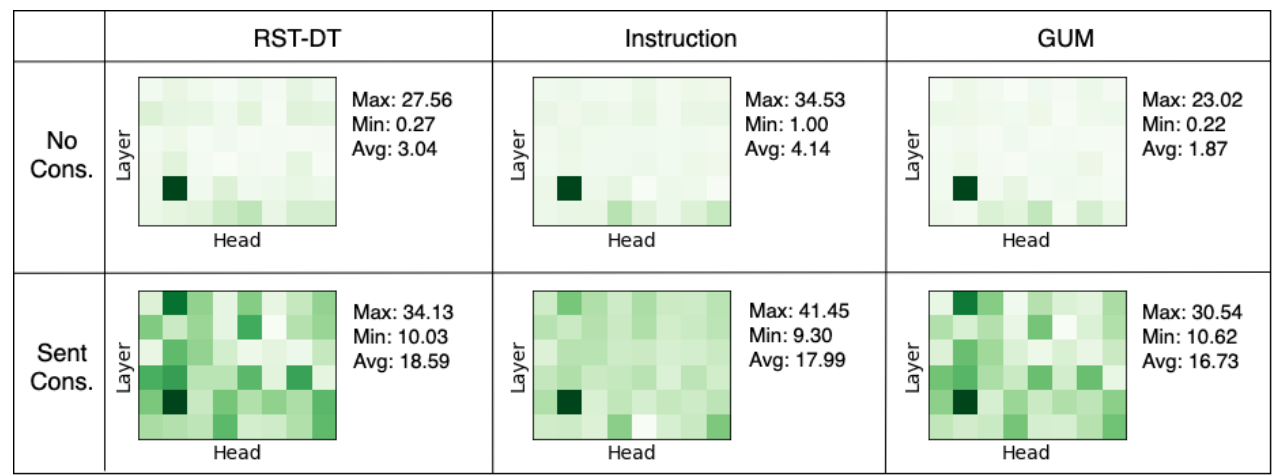

Figure 4: The Unlabeled Attachment Score of trees generated by the attention matrix per head on three datasets under two conditions with the CNNDM-6-8 model.

\subsection{Performance of Heads}

While all previous results rely on the average attention matrices, we now analyze whether discourse information is evenly distributed across attention heads, or if a subset of the heads contains the majority of discourse related information.

We describe this analysis only for CLE for two reasons: (a) the summarization model seemingly captures more dependency-related discourse information than structure information; (b) compared with Eisner, the CLE approach is more flexible, by also covering non-projective dependency trees.

Since the results across all summarization models are consistent, we only show the accuracy heatmap for the $C N N D M-6-8$ model on the three RST-style discourse datasets in Figure 4. Remarkably, for all three datasets, there is one head in the model capturing the vast majority of discourse information, especially in the unconstrained case. Furthermore, the performance of the best single attention head is much better than the one of the average attention matrix shown in section 4.4 (e.g. 34.53 compared to 19.51 on the GUM dataset without sentence constraints). These intriguing findings will be further explored in future work.

\subsection{Analysis of Generated Trees}

Localness of Trees: To further verify that the generated trees are non-trivial, for instance simply connecting adjacent EDUs, we analyze the quality of the trees produced with the second attention head on the second layer, which is the top performer among all the heads shown in Figure 4. First, we separate all dependency relationships into two classes: local, holding between two adjacent EDUs, and distant, including all other relations between non-adjacent EDUs. Then we compute the ratio of the correctly predicted dependencies which are local (Local Ratio Corr.), as well as the

\begin{tabular}{c|c|c} 
Measurement(\%) & No Cons. & Sent Cons. \\
\hline \multicolumn{3}{|c}{ RST-DT } \\
\hline $\begin{array}{c}\text { Local Ratio Corr. } \\
\text { Local Ratio GT }\end{array}$ & 77.78 & 79.17 \\
Local Ratio Ours & 46.52 & 53.22 \\
\hline \multicolumn{3}{c}{ Instruction } \\
\hline Local Ratio Corr. & 81.15 & 84.35 \\
Local Ratio GT & \multicolumn{2}{|c}{59.82} \\
Local Ratio Ours & 47.90 & 60.54 \\
\hline \multicolumn{3}{c}{ GUM } \\
\hline Local Ratio Corr. & 77.99 & 80.20 \\
Local Ratio GT & \multicolumn{2}{|c}{53.28} \\
Local Ratio Ours & 39.97 & 53.76 \\
\hline
\end{tabular}

Table 5: Measurements on the locality of the generated dependency trees, all numbers are in \%. Corr. represents all the correct predictions, GT the ground-truth trees, and Ours the generated tree respectively.

ratio of local dependencies in the generated trees (Local Ratio Ours), and in the ground-truth trees (Local Ratio GT). The results of this analysis are shown in Table 5. For all datasets, the ratio of correctly predicted local dependencies (Local Ratio Corr.) (being $>50$ ) is larger than the ratio for distant relations, which appears reasonable, since local dependency predictions are easier to predict than distant ones. Further, comparing (Local Ratio GT) and (Local Ratio Ours) without the sentence constraint (first column) shows that the number of local dependency relations in the ground-truth discourse trees is consistently larger than the predicted number. This indicates that the discourse information learned in the attention matrices goes beyond the oftentimes predominant local positional information. However, even without the sentence constraint (first column), when the CLE algorithm can predict trees of any form, more than $40 \%$ of the relations are predicted as local, suggesting that the standard CLE approach can already capture local information well.

Adding the sentence constraint (second column), 


\begin{tabular}{c|c|c|c|c|c} 
& Branch & Height & Leaf & Arc & vac. (\%) \\
\hline \multicolumn{6}{c}{ RST-DT } \\
\hline Ours(Sent Cons) & 1.50 & 27.06 & 0.37 & 0.10 & $3 \%$ \\
Ours(No Cons) & 1.74 & 25.76 & 0.49 & 0.12 & $3 \%$ \\
GT Tree & 2.10 & 8.19 & 0.51 & 0.13 & $2 \%$ \\
\hline \multicolumn{6}{c}{ Instruction } \\
\hline Ours(Sent Cons) & 1.56 & 15.74 & 0.39 & 0.13 & $3 \%$ \\
Ours(No Cons) & 1.80 & 14.35 & 0.50 & 0.14 & $3 \%$ \\
GT Tree & 1.59 & 8.49 & 0.41 & 0.15 & $1 \%$ \\
\hline \multicolumn{6}{c}{ GUM } \\
\hline Ours(Sent Cons) & 1.61 & 44.94 & 0.40 & 0.05 & $0 \%$ \\
Ours(No Cons) & 2.14 & 43.08 & 0.54 & 0.08 & $0 \%$ \\
GT Tree & 2.02 & 12.17 & 0.51 & 0.04 & $0 \%$ \\
\hline
\end{tabular}

Table 6: Statistics of our generated trees and the gold standard trees in terms of the average branch width, average height, average leaf ratio (micro), average normalized arc length of the trees and percentage of the Vacuous trees.

we find that the local dependency ratio of the generated trees (Local Ratio Ours) further increases by more than $10 \%$ across all three datasets. This makes intuitive sense, since the sentence constraint forces the generated trees to purely focus on local aspects within each sentence. To sum up, we find that the learned attention matrices contains both local and distant dependency information, although local dependency predictions perform better.

Properties of Trees: Following Ferracane et al. (2019), we structurally inspect the generated dependency trees, and compare them with the gold trees on all three datasets. This comparison is presented in Table 6, showing the average branch width, average height, average leaf ratio (micro) and average normalized arc length of the trees as well as the percentage of vacuous trees in each dataset ${ }^{6}$.

Looking at Table 6, it appears that our tree structure properties are similar to the ground-truth properties in regards to all measures except the height of the tree, which indicates that our trees tend to be generally deeper than gold standard trees, despite having a similar branch width and leaf ratio. Furthermore, our trees are even deeper when using the sentence constraint. Plausibly, by forcing each sentence to have its own sub-tree can make shallower inter-sentential structures less likely. Exploring potential causes for the difference in tree-height, possibly due to the summarization task itself, are left as future work.

\footnotetext{
${ }^{6} \mathrm{~A}$ vacuous tree is a special tree in which the root is one of the first two EDUs, with all nodes are children of the root.
}

\subsection{Additional Results on Model Sensitivity to Initialization and Summarizer Quality}

To investigate whether the performance is consistent cross different random initializations, and to explore the influence of the results with respect to the quality of the summarizer, we perform additional experiments with the 'CNNDM-6-8' model $^{7}$. Overall, we find that the performance is rather similar across random initializations. Interestingly, a single head consistently shows better performance than all other heads across different initialization as well as datasets; however, while the position of the top-performing head is not always the same, it is often located in the second layer of the model. Regarding the second experiment exploring sensitivity to the summarizer quality, we create summarizers of increasing quality by providing more and more training. As expected, we find that as the summarization model is trained for additional steps, more accurate discourse information is learnt, concentrated in a single head.

\section{Conclusions and Future Work}

We present a novel framework to infer discourse trees from the attention matrices learned in a transformer-based summarization model. Experiment across models and datsets indicates that both dependency and structural discourse information are learned, that such information is typically concentrated in a single head, and that the attention matrix also covers long distance discourse dependencies. Overall, consistent results across datasets and models suggest that the learned discourse information is general and transferable inter-domain.

In the future, we want to explore if simpler summarizers like BERTSUM (Liu and Lapata, 2019b) can also capture discourse info; specifically studying if the importance of the heads corresponds to the captured discourse info, which may help pruning summarization model by incorporating discourse info, in spirit of Xiao et al. (2020).

With respect to dependency tree generation possible improvements could come by looking for additional strategies balancing between guidance and flexibility, as Kuhlmann and Nivre (2006) explore for syntactic dependency parsing.

To address the problem of data sparsity in discourse parsing, we want to synergistically leverage other discourse-related tasks, in addition to sentiment and summarization, like topic modeling.

${ }^{7}$ More details can be found in Appendix C. 


\section{Acknowledgments}

We thank the anonymous reviewers and the UBCNLP group for their insightful comments. This research was supported by the Language \& Speech Innovation Lab of Cloud BU, Huawei Technologies Co., Ltd.

We further acknowledge the support of the Natural Sciences and Engineering Research Council of Canada (NSERC).

Nous remercions le Conseil de recherches en sciences naturelles et en génie du Canada (CRSNG) de son soutien.

\section{References}

Lynn Carlson, Mary Ellen Okurowski, and Daniel Marcu. 2002. RST discourse treebank. Linguistic Data Consortium, University of Pennsylvania.

Y. Chu and T. Liu. 1965. On the shortest arborescence of a directed graph.

Arman Cohan, Franck Dernoncourt, Doo Soon Kim, Trung Bui, Seokhwan Kim, Walter Chang, and Nazli Goharian. 2018. A discourse-aware attention model for abstractive summarization of long documents. NAACL HLT 2018 - 2018 Conference of the North American Chapter of the Association for Computational Linguistics: Human Language Technologies - Proceedings of the Conference, 2:615-621.

Jack Edmonds. 1967. Optimum Branchings.

Jason M. Eisner. 1996. Three new probabilistic models for dependency parsing: An exploration. In $C O L$ ING 1996 Volume 1: The 16th International Conference on Computational Linguistics.

Elisa Ferracane, Greg Durrett, Junyi Jessy Li, and Katrin Erk. 2019. Evaluating discourse in structured text representations. arXiv preprint arXiv:1906.01472.

Tsutomu Hirao, Yasuhisa Yoshida, Masaaki Nishino, Norihito Yasuda, and Masaaki Nagata. 2013. SingleDocument Summarization as a Tree Knapsack Problem. Technical report.

Patrick Huber and Giuseppe Carenini. 2019. Predicting discourse structure using distant supervision from sentiment. In Proceedings of the 2019 Conference on Empirical Methods in Natural Language Processing and the 9th International Joint Conference on Natural Language Processing (EMNLPIJCNLP), pages 2306-2316.

Patrick Huber and Giuseppe Carenini. 2020. MEGA RST discourse treebanks with structure and nuclearity from scalable distant sentiment supervision. In Proceedings of the 2020 Conference on Empirical Methods in Natural Language Processing (EMNLP), pages 7442-7457, Online. Association for Computational Linguistics.
Shafiq Joty, Giuseppe Carenini, and Raymond $\mathrm{T}$ Ng. 2015. Codra: A novel discriminative framework for rhetorical analysis. Computational Linguistics, 41(3):385-435.

Dan Jurafsky and James H Martin. 2014. Speech and language processing, volume 3. Pearson London.

Yuta Kikuchi, Tsutomu Hirao, Hiroya Takamura, Manabu Okumura, and Masaaki Nagata. 2014. Single document summarization based on nested tree structure. 52nd Annual Meeting of the Association for Computational Linguistics, ACL 2014 - Proceedings of the Conference, 2:315-320.

Naoki Kobayashi, Tsutomu Hirao, Kengo Nakamura, Hidetaka Kamigaito, Manabu Okumura, and Masaaki Nagata. 2019. Split or merge: Which is better for unsupervised rst parsing? In Proceedings of the 2019 Conference on Empirical Methods in Natural Language Processing and the 9th International Joint Conference on Natural Language Processing (EMNLP-IJCNLP), pages 5801-5806.

Marco Kuhlmann and Joakim Nivre. 2006. Mildly nonprojective dependency structures. In Proceedings of the COLING/ACL 2006 Main Conference Poster Sessions, pages 507-514, Sydney, Australia. Association for Computational Linguistics.

Sujian Li, Liang Wang, Ziqiang Cao, and Wenjie Li. 2014. Text-level discourse dependency parsing. In Proceedings of the 52nd Annual Meeting of the Association for Computational Linguistics (Volume 1: Long Papers), pages 25-35, Baltimore, Maryland. Association for Computational Linguistics.

Nelson F. Liu, Matt Gardner, Yonatan Belinkov, Matthew E. Peters, and Noah A. Smith. 2019a. Linguistic knowledge and transferability of contextual representations. In Proceedings of the 2019 Conference of the North American Chapter of the Association for Computational Linguistics: Human Language Technologies, Volume 1 (Long and Short Papers), pages 1073-1094, Minneapolis, Minnesota. Association for Computational Linguistics.

Yang Liu and Mirella Lapata. 2018. Learning structured text representations. Transactions of the Association for Computational Linguistics, 6:63-75.

Yang Liu and Mirella Lapata. 2019a. Hierarchical transformers for multi-document summarization. In Proceedings of the 57th Annual Meeting of the Association for Computational Linguistics, pages 5070-5081, Florence, Italy. Association for Computational Linguistics.

Yang Liu and Mirella Lapata. 2019b. Text Summarization with Pretrained Encoders. EMNLP-IJCNLP 2019 - 2019 Conference on Empirical Methods in Natural Language Processing and 9th International Joint Conference on Natural Language Processing, Proceedings of the Conference, pages 3730-3740. 
Yang Liu, Ivan Titov, and Mirella Lapata. 2019b. Single document summarization as tree induction. In Proceedings of the 2019 Conference of the North American Chapter of the Association for Computational Linguistics: Human Language Technologies, Volume 1 (Long and Short Papers), pages 1745-1755, Minneapolis, Minnesota. Association for Computational Linguistics.

William C. Mann and Sandra A. Thompson. 1988. Rhetorical Structure Theory: Toward a functional theory of text organization.

Daniel Marcu. 1999. Discourse Trees are Good Indicators of Importance in Text. Advances in Automatic Text Summarization, pages 123-136.

David Mareček and Rudolf Rosa. 2019. From balustrades to pierre vinken: Looking for syntax in transformer self-attentions. In Proceedings of the 2019 ACL Workshop BlackboxNLP: Analyzing and Interpreting Neural Networks for NLP, pages 263275, Florence, Italy. Association for Computational Linguistics.

Ryan McDonald. 2007. A study of global inference algorithms in multi-document summarization. In Proceedings of the 29th European Conference on IR Research, ECIR'07, page 557-564, Berlin, Heidelberg. Springer-Verlag.

Ramesh Nallapati, Bowen Zhou, Cicero dos Santos, Çağlar Guİłlçehre, and Bing Xiang. 2016. Abstractive text summarization using sequence-to-sequence RNNs and beyond. In Proceedings of The 20th SIGNLL Conference on Computational Natural Language Learning, pages 280-290, Berlin, Germany. Association for Computational Linguistics.

Noriki Nishida and Hideki Nakayama. 2020. Unsupervised discourse constituency parsing using viterbi em. Transactions of the Association for Computational Linguistics, 8:215-230.

Alessandro Raganato and Jörg Tiedemann. 2018. An analysis of encoder representations in transformerbased machine translation. In Proceedings of the 2018 EMNLP Workshop BlackboxNLP: Analyzing and Interpreting Neural Networks for NLP, pages 287-297, Brussels, Belgium. Association for Computational Linguistics.

Evan Sandhaus. 2008. The new york times annotated corpus.

Rajen Subba and Barbara Di Eugenio. 2009. An effective discourse parser that uses rich linguistic information. In Proceedings of Human Language Technologies: The 2009 Annual Conference of the North American Chapter of the Association for Computational Linguistics, pages 566-574. Association for Computational Linguistics.

Ashish Vaswani, Noam Shazeer, Niki Parmar, Jakob Uszkoreit, Llion Jones, Aidan N. Gomez, Łukasz
Kaiser, and Illia Polosukhin. 2017. Attention is all you need. Advances in Neural Information Processing Systems, 2017-Decem(Nips):5999-6009.

Chenguang Wang, Xiao Liu, and Dawn Song. 2020. Language models are open knowledge graphs.

Yizhong Wang, Sujian Li, and Houfeng Wang. 2017. A two-stage parsing method for text-level discourse analysis. In Proceedings of the 55th Annual Meeting of the Association for Computational Linguistics (Volume 2: Short Papers), pages 184-188.

Zhiyong Wu, Yun Chen, Ben Kao, and Qun Liu. 2020. Perturbed masking: Parameter-free probing for analyzing and interpreting BERT. In Proceedings of the 58th Annual Meeting of the Association for Computational Linguistics, pages 4166-4176, Online. Association for Computational Linguistics.

Wen Xiao and Giuseppe Carenini. 2019. Extractive summarization of long documents by combining global and local context. In Proceedings of the 2019 Conference on Empirical Methods in Natural Language Processing and the 9th International Joint Conference on Natural Language Processing (EMNLP-IJCNLP), pages 3011-3021, Hong Kong, China. Association for Computational Linguistics.

Wen Xiao, Patrick Huber, and Giuseppe Carenini. 2020. Do we really need that many parameters in transformer for extractive summarization? discourse can help! In Proceedings of the First Workshop on Computational Approaches to Discourse, pages 124-134, Online. Association for Computational Linguistics.

Jiacheng $\mathrm{Xu}$, Zhe Gan, Yu Cheng, and Jingjing Liu. 2020. Discourse-aware neural extractive text summarization. In Proceedings of the 58th Annual Meeting of the Association for Computational Linguistics, pages 5021-5031, Online. Association for Computational Linguistics.

Nan Yu, Meishan Zhang, and Guohong Fu. 2018. Transition-based neural rst parsing with implicit syntax features. In Proceedings of the 27th International Conference on Computational Linguistics, pages 559-570.

Amir Zeldes. 2017. The GUM corpus: Creating multilayer resources in the classroom. Language Resources and Evaluation, 51(3):581-612.

Xingxing Zhang, Furu Wei, and Ming Zhou. 2019. HIBERT: Document level pre-training of hierarchical bidirectional transformers for document summarization. In Proceedings of the 57th Annual Meeting of the Association for Computational Linguistics, pages 5059-5069, Florence, Italy. Association for Computational Linguistics. 


\section{A Performance of the Summarization Task}

Table. 7 shows the performance of different summarization models. In general, adding additional layers and heads does not consistently increase the performance on the summarization task itself.

\begin{tabular}{c|c|c|c|c|c} 
Dataset & \#Layer & \#Head & R-1 & R-2 & R-L \\
\hline CNNDM & 2 & 1 & 40.92 & 18.69 & 37.85 \\
CNNDM & 2 & 8 & 41.02 & 18.78 & 37.96 \\
CNNDM & 6 & 8 & 41.03 & 18.69 & 37.86 \\
\hline NYT & 2 & 1 & 43.64 & 25.58 & 36.87 \\
NYT & 2 & 8 & 44.11 & 26.08 & 37.34 \\
NYT & 6 & 8 & 43.93 & 25.99 & 37.15
\end{tabular}

Table 7: The in-domain performance of the summarizers.

\section{B Full Results on Overall Tree Parsing}

We show the overall results of all the six summarization models on constituency/dependency parsing in Table.8, the results of three of them are shown in Table.2, Table. 3 and Table. 4 in the main paper.

\section{Results on Sensitivity to Initialization and Summarizer Quality}

To explore if the models with different random initialization have consistent performances, we train 5 models with 6 layers and 8 heads on the CNNDM dataset with different initialization, and the results of each layer for constituency/dependency parsing are shown in Table.9. We can find that the results are relatively consistent across different initialization, and additional exploration on the performance of all the heads (Fig.5) show that, with different initialization of the model, there is consistently one head containing most of the discourse information, but the position of that head is not fixed.

We further do the experiments on dependency parsing during training the summarizer, to see how the performance changes as the summarizer become better, and show the max and mean UAS over three datasets for all attention heads in the 'cnndm-6-8' model by the CLE algorithms after training for $(0,1 \mathrm{k}, 5 \mathrm{k}, 10 \mathrm{k}, 20 \mathrm{k})$ steps in Fig.6. We also show the heatmaps of the average UAS across three datasets for all the heads in Fig.7. We can find that as the summarizer is trained for more steps, more discourse information is learned, and it's more concentrated in one head. Interestingly, the mean UAS of dependency trees generated by CLE algorithm with sentence constraints show a different trend, which may due to the concentration of the discourse information on single head as the model trained for more steps, as it shows in Figure.7. 


\begin{tabular}{|c|c|c|c|c|c|c|}
\hline \multirow{2}{*}{ Model } & \multicolumn{2}{|c|}{ Const } & \multicolumn{2}{|c|}{ Eisner } & \multicolumn{2}{|c|}{ CLE } \\
\hline & No Cons. & Sent Cons. & No Cons. & Sent Cons. & No Cons. & Sent Cons. \\
\hline & \multicolumn{6}{|c|}{ RSTDT } \\
\hline CNNDM-2-1 & $61.16 / 59.67$ & $76.23 / 74.63$ & $\mathbf{2 3 . 6 5} / 4.80$ & $\mathbf{2 8 . 2 4} / 18.23$ & $\mathbf{2 1 . 5 6} / 1.45$ & $\mathbf{2 9 . 2 9} / 19.56$ \\
\hline CNNDM-2-8 & $62.65 / 59.75$ & $76.42 / 74.28$ & $22.09 / 8.40$ & $26.23 / 21.29$ & $20.31 / 6.13$ & $26.57 / 22.67$ \\
\hline CNNDM-6-8 & $60.33 / 60.79$ & $75.44 / 75.04$ & $7.89 / 20.48$ & $13.83 / 27.78$ & $7.28 / 17.30$ & $16.10 / 28.50$ \\
\hline NYT-2-1 & $60.27 / 60.23$ & $75.57 / 75.29$ & $9.76 / 14.84$ & $23.18 / 20.61$ & $6.18 / 12.68$ & $21.06 / 21.73$ \\
\hline NYT-2-8 & $\mathbf{6 3 . 2 0} / 59.65$ & $76.63 / 75.23$ & $7.35 / 9.74$ & $16.04 / 21.27$ & $6.44 / 7.09$ & $16.72 / 22.90$ \\
\hline NYT-6-8 & $62.42 / 62.17$ & $\mathbf{7 6 . 6 5} / 75.58$ & $15.74 / 12.51$ & $24.30 / 18.90$ & $13.71 / 10.59$ & $25.04 / 21.14$ \\
\hline \multirow[t]{2}{*}{ Random } & $58.60(0.1)$ & $74.10(0.1)$ & $11.16(0.2)$ & $20.28(0.2)$ & $1.67(0.08)$ & $18.72(0.11)$ \\
\hline & \multicolumn{6}{|c|}{ Instruction } \\
\hline CNNDM-2-1 & $61.06 / 59.84$ & $71.39 / 70.29$ & $\mathbf{3 1 . 0 7} / 4.39$ & $29.33 / 13.45$ & $\mathbf{2 8 . 0 6} / 2.08$ & $\mathbf{3 7 . 3 8} / 18.12$ \\
\hline CNNDM-2-8 & $61.44 / 60.55$ & $71.13 / 71.09$ & $26.98 / 8.89$ & $24.72 / 14.75$ & $24.56 / 5.23$ & $28.70 / 20.58$ \\
\hline CNNDM-6-8 & $60.32 / 61.22$ & $71.24 / 70.88$ & $8.53 / 19.51$ & $9.93 / 21.96$ & $6.92 / 15.85$ & $14.93 / 25.78$ \\
\hline NYT-2-1 & $60.31 / 61.30$ & $71.40 / \mathbf{7 1 . 4 3}$ & $10.67 / 21.15$ & $18.99 / 21.19$ & $7.82 / 17.59$ & $21.30 / 24.54$ \\
\hline NYT-2-8 & $61.27 / 60.51$ & $70.80 / 70.90$ & $6.26 / 12.59$ & 13.64 / 19.34 & $5.25 / 7.96$ & $13.39 / 21.92$ \\
\hline NYT-6-8 & 61.32 / 61.27 & $71.30 / 70.03$ & $16.22 / 12.14$ & $22.79 / 16.37$ & $14.81 / 9.81$ & $25.44 / 21.10$ \\
\hline \multirow[t]{2}{*}{ Random } & $59.49(0.3)$ & $70.53(0.1)$ & $13.14(0.33)$ & $19.31(0.44)$ & $2.94(0.24)$ & $17.88(0.42)$ \\
\hline & \multicolumn{6}{|c|}{ GUM } \\
\hline CNNDM-2-1 & $58.74 / 57.69$ & $\mathbf{7 2 . 7 3} / 71.92$ & $\mathbf{2 1 . 2 8} / 2.24$ & $\mathbf{2 7 . 2 6} / 16.12$ & $\mathbf{1 9 . 5 0} / 0.70$ & $28.77 / 17.92$ \\
\hline CNNDM-2-8 & $59.98 / 58.43$ & $72.69 / 71.95$ & $19.45 / 4.98$ & $25.00 / 19.25$ & $18.03 / 2.92$ & $25.07 / 20.40$ \\
\hline CNNDM-6-8 & $58.92 / 59.30$ & $72.40 / 72.69$ & $4.74 / 15.80$ & $11.53 / 24.79$ & $4.01 / 13.14$ & $14.85 / 25.37$ \\
\hline NYT-2-1 & $57.81 / 58.84$ & $71.95 / 72.23$ & $5.64 / 12.84$ & $19.94 / 20.19$ & $2.92 / 9.79$ & $18.23 / 19.68$ \\
\hline NYT-2-8 & $\mathbf{6 0 . 1 7} / 58.22$ & $71.98 / 71.82$ & $5.66 / 7.22$ & $15.21 / 18.81$ & 4.54 / 3.96 & $15.25 / 19.31$ \\
\hline NYT-6-8 & $59.62 / 59.25$ & $72.19 / 71.56$ & $12.58 / 9.61$ & $23.35 / 17.14$ & $10.67 / 8.23$ & 22.99 / 19.53 \\
\hline Random & $57.47(0.1)$ & $71.50(0.2)$ & $10.37(0.23)$ & $19.15(0.26)$ & $0.92(0.05)$ & $17.01(0.2)$ \\
\hline
\end{tabular}

Table 8: The RST Parseval Scores of generated constituency trees, Unlabeled Attachment Score of generated dependency trees by Eisner algorithm and CLE algorithm on the three datasets. The numbers in each cell are represented as the performance of (Layer 0 / Layer 1) the results of Random are obtained by applying the parser on random generated matrices for 10 times, and are represented as 'Average (Std)'.

\begin{tabular}{c|c|c|c|c|c|c|c}
\hline \multirow{2}{*}{ Model } & \multicolumn{2}{|c|}{ Const } & \multicolumn{2}{c|}{ Eisner } & \multicolumn{2}{c}{ CLE } \\
& No Cons. & Sent Cons. & No Cons. & Sent Cons. & No Cons. & Sent Cons. \\
\hline & \multicolumn{7}{|c}{ RSTDT } \\
\hline \multirow{2}{*}{ CNNDM-6-8 } & $61.13 / 61.63$ & $75.81 / 75.41$ & $10.32 / 20.99$ & $16.42 / 27.08$ & $9.40 / 18.16$ & $18.89 / 28.33$ \\
& $(1.11) /(1.35)$ & $(0.26) /(0.34)$ & $(4.03) /(2.80)$ & $(3.62) /(1.37)$ & $(3.92) /(3.25)$ & $(4.19) /(1.59)$ \\
\hline Random & $58.6(0.1)$ & $74.10(0.1)$ & $11.16(0.2)$ & $20.28(0.2)$ & $1.67(0.08)$ & $18.72(0.11)$ \\
\hline \multicolumn{7}{|c|}{ Instruction } \\
\hline \multirow{2}{*}{ CNNDM-6-8 8} & $61.87 / 61.06$ & $70.84 / 70.94$ & $11.50 / 19.78$ & $12.91 / 22.45$ & $9.79 / 16.53$ & $17.81 / 26.30$ \\
& $(1.17) /(0.98)$ & $(0.51) /(0.34)$ & $(5.71) /(2.17)$ & $(4.39) /(1.48)$ & $(5.31) /(2.73)$ & $(4.70) /(1.64)$ \\
\hline Random & $59.49(0.3)$ & $70.53(0.1)$ & $13.14(0.33)$ & $19.31(0.44)$ & $2.94(0.24)$ & $17.88(0.42)$ \\
\hline \multirow{7}{*}{ CNNDM-6-8 } & $58.19 / 58.71$ & $72.28 / 72.48$ & $7.62 / 15.69$ & $14.62 / 24.02$ & $6.77 / 13.23$ & $17.32 / 25.13$ \\
& $(0.82) /(0.97)$ & $(0.27) /(0.16)$ & $(3.97) /(2.87)$ & $(3.77) /(1.36)$ & $(3.96) /(3.29)$ & $(3.85) /(1.22)$ \\
\hline Random & $57.47(0.1)$ & $71.50(0.2)$ & $10.37(0.23)$ & $19.15(0.26)$ & $0.92(0.05)$ & $17.01(0.2)$ \\
\hline
\end{tabular}

Table 9: The average RST Parseval Scores of generated constituency trees, the average Unlabeled Attachment Scores of generated dependency trees by the Eisner and CLE algorithms, on the three datasets with 5 random initialization, the numbers in parenthesis are the standard deviation across different run 


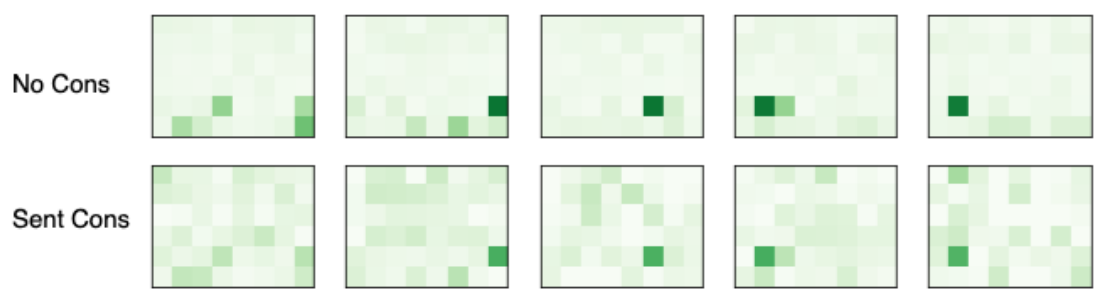

Figure 5: The heatmap of average UAS across three discourse datasets for all attention heads in the models with different initialization by the CLE algorithm.

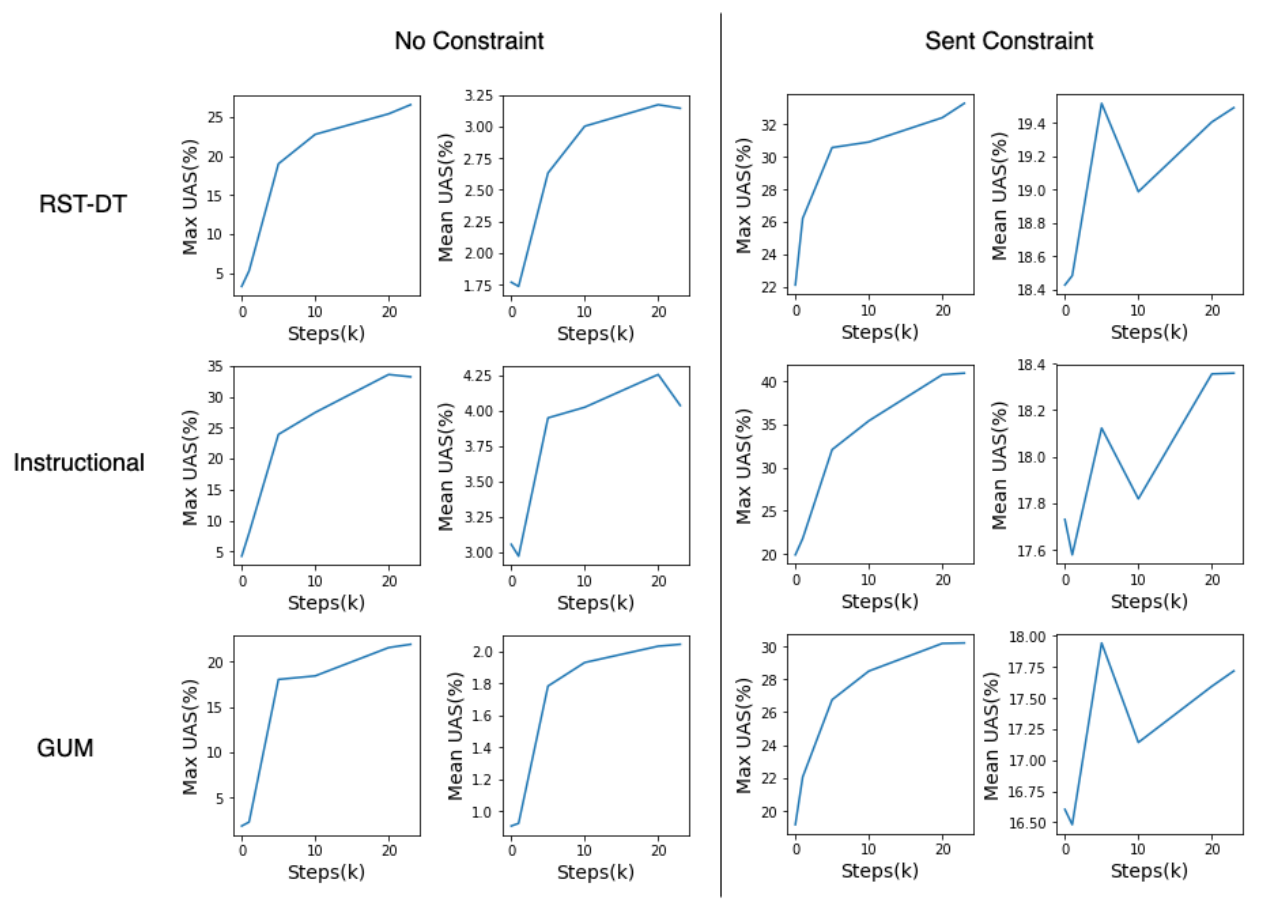

Figure 6: Max and Mean UAS of dependency trees generated by CLE algorithm on all attention heads (48) of the model 'cnndm-6-8', after training for $(0,1,5,10,20,23) \mathrm{K}$ steps on RST-DT(top), Instructional(middle) and GUM(bottom) datasets. The corresponding ROUGE scores are increasing.

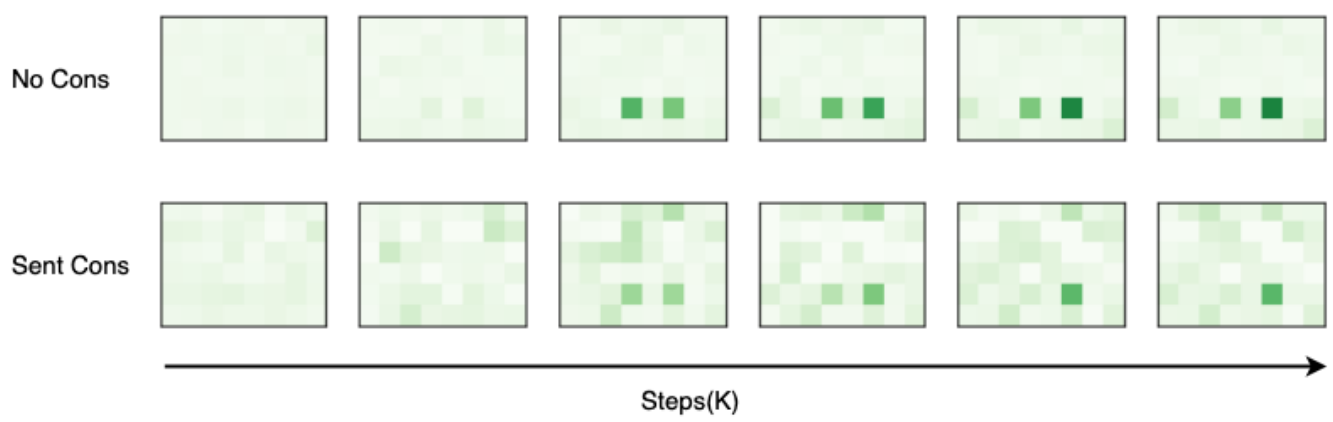

Figure 7: The heatmaps of the average UAS across the three discourse datasets for all the heads during training the summarization model. 